\title{
Control across scales by positive and negative feedlback
}

\section{R. Sepulchre, ${ }^{1}$ G. Drion, ${ }^{2}$ and A. Franci ${ }^{3}$}

1 Department of Engineering, University of Cambridge, Trumpington Street, Cambridge CB2 1PZ r.sepulchre@eng.cam.ac.uk

2 Department of Electrical Engineering and Computer Science, University of Liege, Liege, Belgium. gdrion@uliege.be

${ }^{3}$ National Autonomous University of Mexico, Science Faculty, Department of Mathematics, Coyoacán, D.F. México. afranci@ciencias.unam.mx

Xxxx. Xxx. Xxx. Xxx. YYYY. AA:1-26

https://doi.org/10.1146/((please add article doi))

Copyright (C) YYYY by Annual Reviews. All rights reserved

\section{Keywords}

feedback control, excitability, ultra-sensitivity, positive and negative feedback, neuronal networks, multi-scale control.

\section{Abstract}

Feedback is a key element of regulation, as it shapes the sensitivity of a process to its environment. Positive feedback up-regulates, negative feedback down-regulates. Many regulatory processes involve a mixture of both, whether in nature or in engineering. This paper revisits the mixed feedback paradigm, with the aim of investigating control across scales. We propose that mixed feedback regulates excitability and that excitability plays a central role in multi-scale neuronal signaling. We analyze this role in a multi-scale network architecture inspired from neurophysiology. The nodal behavior defines a meso-scale that connects actuation at the micro-scale to regulation at the macro-scale. We show that mixed-feedback nodal control provides regulatory principles at the network scale, with a nodal resolution. In this sense, the mixed feedback paradigm is a control principle across scales. 


\section{Contents}

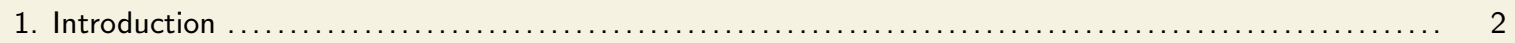

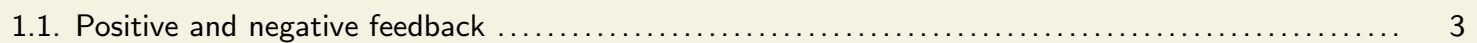

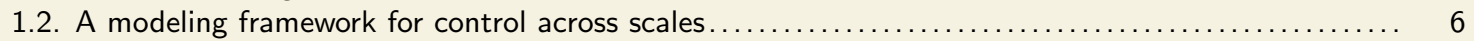

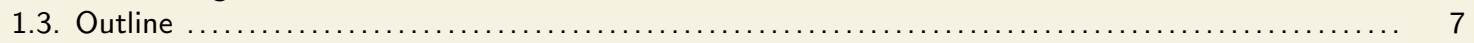

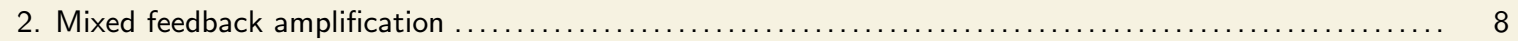

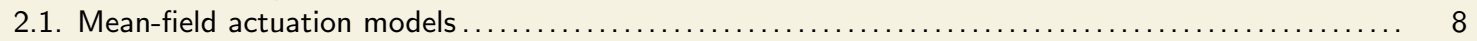

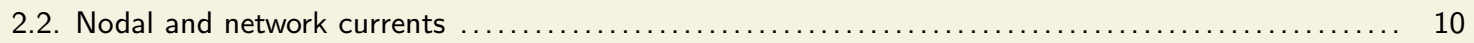

2.3. Excitability: a mixed feedback principle for signaling across scales $\ldots \ldots \ldots \ldots \ldots \ldots \ldots \ldots \ldots \ldots \ldots, 11$

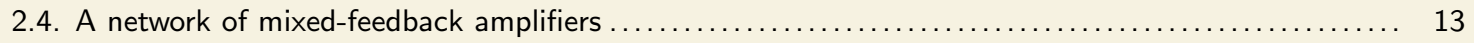

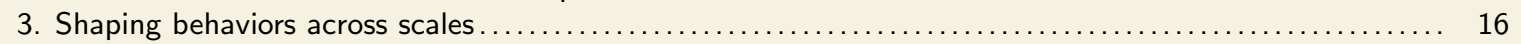

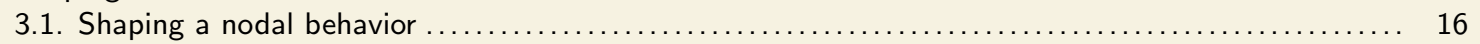

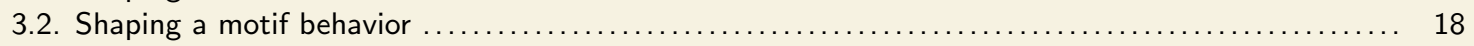

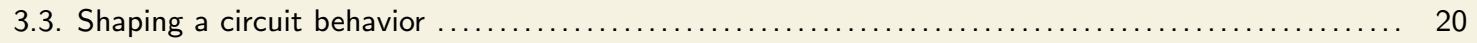

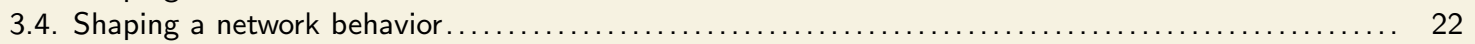

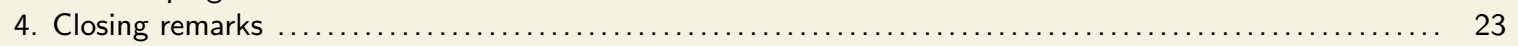

\section{Introduction}

Control systems are increasingly multi-scale. Cheaper sensing, cheaper actuation, and cheaper data processing motivate control algorithms that feed back signals at a microscale to regulate an objective at a macro-scale. Emblematic examples include traffic control (a population of individually controlled cars determines the traffic of a road or a city), soft robotics (a population of micro-actuators determines the behavior of a robot), and tissue engineering (a population of regulated cells determines the properties of a tissue). The present paper is motivated by the brain control of neuronal circuits, which involves reshaping the spatio-temporal electrical activity of neuronal populations across a formidable range of scales. What is common to all the examples above is that variables at a macro-scale are regulated by myriads of actuators at a micro-scale.

The increasing disparity of scales requires novel feedback control paradigms. In classical control, the distinction between noise and signal is based on a separation of scales in amplitude and frequency. The noisy part of a signal is its small-scale component. This distinction must be reconsidered when the signal range of a single micro-scale actuator or sensor is well into the noise range of the macro-scale regulation objective.

In response to the multi-scale challenge, the last two decades have witnessed a particular focus on distributed control paradigms (see e.g. (1) and references therein). In distributed control, the global objective at the macro-scale is reformulated as a local objective at the micro-scale. Local feedback loops are designed at the micro-scale and the local objective is made global through diffusive mechanisms such as consensus or synchronisation (2). This approach works best when the macro-scale is homogeneous, that is, the local control problem is the same everywhere, or, equivalently, space and time invariant. Even in the situation of a perfectly homogeneous control problem, the distributed paradigm faces limitations inherent to the disparity of scales between the micro-scale of sensing and actuation and the macro-scale of regulation, see e.g. (3).

In heterogenous multi-scale problems, the prevalent control architecture is hierarchical. The control problem at the macro-scale is decomposed into a sequence of nested control

2 Sepulchre, Drion, Franci 
problems at decreasing meso-scales. The amplitude, temporal, and spatial scales all obey the same hierarchy: signals of small amplitude and high frequency are modeled and controlled at finer scales, whereas signals of larger amplitude at lower frequencies are modeled and controlled at larger scales. This nested architecture allows for a nested design of regulatory feedback loops. Outer feedback loops regulate coarser scales while inner feedback loops regulate finer scales. The nested feedback loops minimally interact with each other because they deal with distinct scales. Such control architectures are common in engineering and widespread in complex control systems. However, hierarchical constraints limit performance: outer control loops are constrained to low-frequency signals, which makes them sluggish with respect to the inner scales. Inner control loops are constrained to high-frequency signals, which makes them noisy with respect to the outer scales. This performance limitation becomes problematic as the disparity of scales increases. It is perhaps the most evident difference between the performance of robots and animals in comparable tasks. Feedback regulation in biological systems does not seem constrained by a hierarchy of scales. Feedback regulation seems to occur across scales at the temporal and spatial resolution of the tiny. Feedback loops appear interlocked at all scales rather than nested from the tiny to the large. We still lack a theory to analyze and design artificial control systems with such capabilities.

Inspired by the physiology of neuronal circuits, we investigate the role of feedback at a cellular scale in shaping the spatio-temporal behavior of a neuronal network across scales. We revisit the central biophysical model of cellular excitability as a particular regulation mechanism that requires both positive and negative feedback. We propose that this mixed feedback mechanism enables signaling across scales by converting specific tiny continuous variations of an input signal to discrete events that signal to specific larger scales. We provide several examples of neurophysiological relevance where sensitivity at the circuit or network scale is shaped by feedback regulation of excitability at a cellular level. At an abstract level, our objective is to contrast the role of nodal control and the role of network control. Here nodal control refers to modulating the nodal behavior whereas network control refers to modulating the nodal interconnections, that is, the network topology. In neurophysiology, nodal control refers to the modulation of cellular excitability via the modulation of specific intrinsic ionic conductances; network control refers to the modulation of synaptic transmission via the control of synaptic conductances. In artificial neural networks, much emphasis has been placed on network control, and the learning performance of feedforward networks via network control. Those models often neglect nodal control and neuronal excitability. Our paper has a complementary focus: we model nodal excitability and investigate how feedback regulation at a nodal scale can shape a network behavior even with a fixed interconnection structure. Our illustrations suggest that nodal control is not subject to the performance limitation of nested feedback architectures and that the nodal control of excitability is a regulation principle across scales.

\subsection{Positive and negative feedback}

A feedback system is a system that admits the block-diagram representation in Figure 1. It implies an input-output model of the open-loop system, that is, the system without feedback. The open-loop system is modeled as a an operator that transforms input signals into output signals. The feedback loop models the influence of the (past) output on the (future) input. Feedback modeling is central to control theory because feedback is an essential 


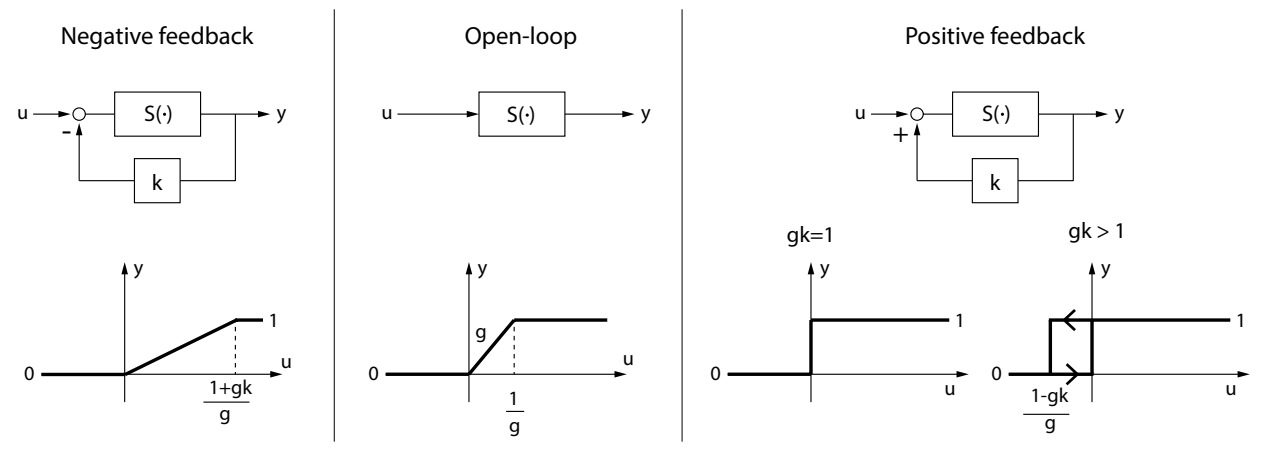

Figure 1

a. An open-loop input-output process (center), a positive feedback system (right), and a negative feedback system (left). b. The distinct roles of positive and negative feedback around a memoryless saturating process. Negative feedback down-regulates and broadens the linear range of the process. Positive feedback make the feedback system ultra-sensitive, promotes a discrete output readout, and creates hysteresis (memory). Mixed feedback is sufficient to modulate a process between three distinct types of behavior : linear continuous, ultra-sensitive, and hysteretic discrete.

component of regulation. Feedback can up-regulate or down-regulate a process. We refer to up-regulation as positive feedback and down-regulation as negative feedback. Positive feedback increases the sensitivity of the output to an input perturbation: the feedback loop amplifies the disturbance and acts as a destabilizing mechanism for the process. Negative feedback decreases the sensitivity of the output to an input perturbation: the feedback counteracts the disturbance and acts as a stabilizing mechanism for the process.

Some key features of feedback can be understood from the elementary saturating static model

$$
y=\operatorname{sat}_{g}(u)=\left\{\begin{array}{c}
0, u \leq 0 \\
g v, 0 \leq u \leq \frac{1}{g} \\
1, u \geq \frac{1}{g}
\end{array}\right.
$$

in the feedback configuation shown in Figure 1. The parameter $g$ is called the open-loop gain and the parameter $k$ is called the feedback gain. The open-loop model is $y=\operatorname{sat}_{g}(u)$. It is a linear model with gain $\frac{\delta y}{\delta u}=g$ over the restricted range $u \in\left[0, \frac{1}{g}\right]$. Away from this linear range, the process saturates and the output is insensitive to input variations. The closed-loop model is defined by the implicit relationship $y=\operatorname{sat}_{g}( \pm k y+u)$. In a negative feedback configuration, the closed-loop model rewrites as $y=\operatorname{sat}_{g^{\prime}}(u)$ with $g^{\prime}=\frac{g}{1+g k}$. The closed-loop model is like the open-loop model, but with a lower gain over a broader linear range. Instead, in a positive feedback configuration, the linear range decreases and the gain increases. The linear range shrinks to zero for the critical value $k=\frac{1}{g}$, making the behavior ultra-sensitive. For larger positive feedback gains $k>\frac{1}{g}$, the closed-loop model is multivalued over the range $u \in\left[\frac{1}{g}-k, 0\right]$. The output has then a binary readout for every value of the input. A continuous variation of the input signal leads to discontinuous jumps of the output signal between 0 and 1, with hysteresis. Positive feedback has converted an open-loop memoryless process into a closed-loop binary memory.

In spite of its simplicity, the above model captures general features of positive and negative feedback regulation:

4 Sepulchre, Drion, Franci 
- Feedback shapes sensitivity. It alters how a process interacts with its environment, that is, how variations of the input signal affect the output signal.

- Negative feedback makes the output less sensitive to input variations and increases the range of linear behavior. For large values of the open-loop gain $g$, the closed-loop gain $g^{\prime}=\frac{1}{g^{-1}+k} \approx \frac{1}{k}$ becomes almost independent of the open-loop gain, making the closed-loop process robust to unmodeled variations of the open-loop gain.

- Positive feedback makes the output more sensitive to input variations and can be tuned to make the process ultrasensitive.

- Beyond a critical gain, a positive feedback system exhibits memory and hysteresis.

- Saturating processes have a continuous or discrete readout. Negative feedback promotes the continuous readout, whereas positive feedback promotes the discrete readout.

The properties above have a general conceptual value but assume a static model of the process. When a process is dynamic, its gain is not only a function of the input amplitude but also of the input history. It is the attempt to conceptualize the properties of feedback in dynamical models that led to the development of feedback theory. Early developments in engineering were driven by the use of feedback in audio and electronic amplifiers. In the early days of electrical signal transmission, engineers first concentrated on the potential of positive feedback to regenerate signals or to design oscillators (4). The potential of negative feedback to make a device robust to uncertainty was discovered much later, with the invention of the negative feedback amplifier (5). From that time on, the use of negative feedback became much more prominent in engineering than the use of positive feedback. Both the success of negative feedback and the rise of digital technology wiped out the interest of engineers for positive feedback. The situation is different in biology, where both positive and negative feedback have been continuously reported to play a prominent role in regulation. Homeostasis has been a central focus since the early days of physiology, with an emphasis on negative feedback regulation. In the sixties, autocatalysis, a positive feedback mechanism, was discovered to be a fundamental element of gene expression regulation (6). More recently, the role of interlocked positive and negative feedback loops has been repeatedly emphasized in systems biology, see e.g. $(7,8,9)$.

The success of feedback control theory owes much to the fact that many properties of negative feedback systems are well captured with a linear model (10). Following the invention of the negative feedback amplifier, Bell Labs engineers developed the frequencydomain analysis of feedback systems. Linear-time invariant systems can be represented by their frequency response (the Fourier transform of their impulse response), which amounts to modeling the process with a frequency-dependent complex gain. A central insight to the stability theory of feedback systems came from the work of Nyquist (11), who studied the critical frequency at which the loop gain of a process changes sign, turning a negative feedback loop into a positive feedback loop. Modern linear robust control can be regarded as a theory of negative feedback. The feedback controller is designed to ensure equilibrium stability of the closed-loop system, to increase the robustness of the process against model uncertainties, to decrease its sensitivity to external disturbances, and to increase the range of input signals over which the process behaves linearly. Those properties are achieved by shaping the loop gain of the feedback system as a function of frequency.

The elementary example in Figure 1 illustrates that positive feedback is the source of nonlinear phenomena (such as ultra-sensitivity or hysteresis) and hence requires different 
analysis tools. For this reason, the mathematical theory of positive feedback lags far behind the mathematical theory of negative feedback. In this paper, we will concentrate on a very specific combination of positive and negative feedback that is amenable to mathematical analysis. Our interest is in the ability of mixed-feedback to regulate ultra-sensitivity and to shape the discrete readout of a continuous process. Our approach is close in spirit to the loop shaping paradigm of classical control theory. But to cope with the nonlinearity of the feedback system, the loop gain is shaped as a function of amplitude and time, rather than as a function of frequency.

\subsection{A modeling framework for control across scales}

Inspired by the neurophysiology of neuronal circuits, we focus in this paper on electrical behaviors. We consider a multi-scale control problem defined by three distinct scales (Figure 2): a micro-scale of actuation; a macro-scale of regulation; and a behavioral meso-scale that connects the micro and macro scales by dynamical relationships between voltages and currents.

The macro-scale of regulation is a spatio-temporal electrical field $V(x, t)$, where $x$ denotes the spatial variable and $t$ denotes the temporal variable.

The meso-scale is defined by a spatial discretization of the electrical field, leading to a network of $N$ nodes with temporal voltage $V_{i}(t), i=1, \ldots, N$. The dynamical behavior of a nodal voltage is determined by the laws of circuit theory. In the language of circuit theory, a node is a connector, that is, a multi-terminal isopotential circuit element determined by Kirchhoff law that all currents into the node sum to zero. In this paper, any current $I$ into a node at potential $V$ is assumed to obey the Ohmic law

$$
I=g(\cdot)(V-\tilde{V})
$$

The current results from a difference of potential with another terminal at potential $\tilde{V}$ across a circuit element of conductance $g(\cdot)$. The notation $g(\cdot)$ stresses that the conductance is not constant. It is shaped by the the micro-scale of actuation. The mean-field of many actuators at the micro-scale is modeled as a dependence of the conductances on the potential field (Figure 2 left).

It is the spatial resolution of a node that defines the meso-scale. A node is isopotential. It determines a resolution of the electrical field at which differences of potentials are neglected. The meso-scale is only constrained to be sufficiently separated from the micro-scale. It must involve a sufficiently large population of actuators at the micro-scale to justify the mean-field model of the conductance.

Currents and voltages play a distinct role in a multi-scale model. Voltage is a variable across while current is a variable into. Variables into are additive. They add to zero at a node. Currents at broader scales are sums of currents at finer scales. Variables across are intensive, i.e., independent of size. A potential is independent of the spatial scale. A difference of potential can be measured between any two terminals. Relationships between across and into variables are inherent to multi-scale modeling. They relate voltages and currents in electricity, or forces and velocities in mechanics.

While this paper focuses on electrical behaviors, that is, relationships between currents and voltages, the multi-scale nature of our model is general. For instance, a multi-scale traffic model can be conceived analogously: in a traffic problem, the variable into is the traffic flow, whereas the variable across is the traffic mass. The analog of the Ohmic law (2)

6 Sepulchre, Drion, Franci 

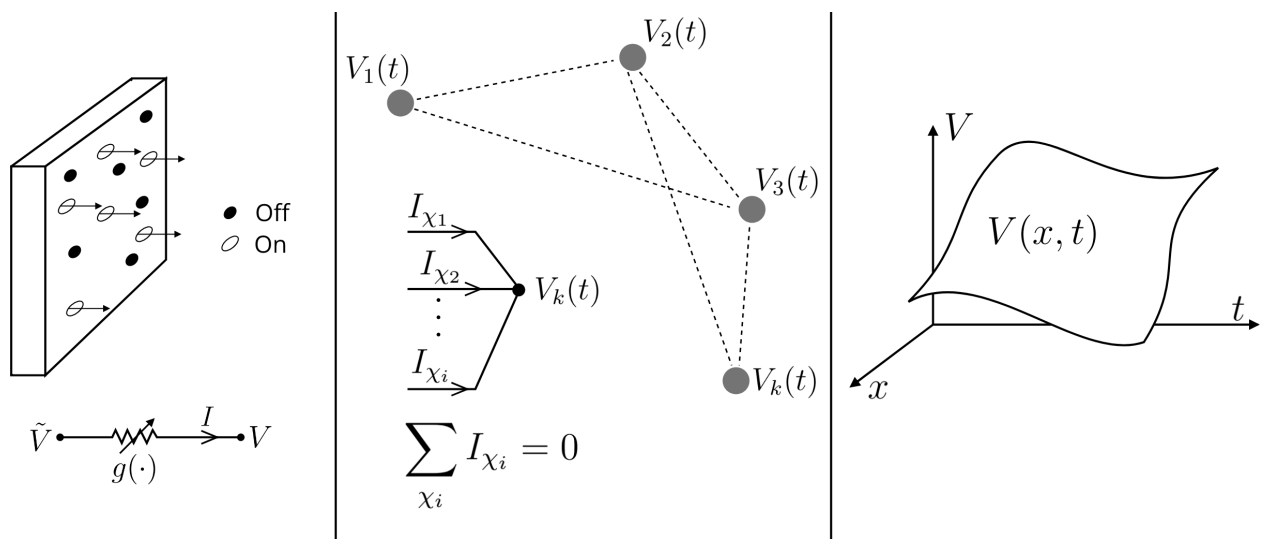

Figure 2

An electrical field modeled at three distinct scales. Regulation macro-scale (right): a controlled spatio-temporal electrical field $V(x, t)$. Behavioral meso-scale (center) : spatial discretization of the electrical field leads to a discrete network of interconnected isopotential nodes $V_{i}(t)$. The nodal behavior is determined by Kirchhoff laws of electrical circuits : currents $I_{\chi_{i}}$ into node $k$ sum to zero. Actuation micro-scale (left): each meso-scale current is modelled as a Ohmic current. The voltage dependence of the conductance models a mean-field control of the current by a population of discrete (on-off) micro-actuators.

is a relationship expressing traffic flow as the product of traffic mass and speed, the analog of conductance. Micro-scale actuation would refer the modulation of speed at a micro-scale, for instance through traffic lights, whereas the macro-scale would be the traffic state of a road or a city.

The proposed multi-scale model is directly inspired from the conductance-based modeling principles of neurophysiology (12). The meso-scale is the scale of cellular neurophysiology. The natural resolution of a node is a single neuron. But a node can also be a patch of a neuron, at a finer spatial scale, or a homogeneous population of neurons, at a coarser scale. A node is modeled with a lumped nodal voltage $V(t)$. Each current into the node models a distinct flow of ions through the cellular membrane. The micro-scale is the scale of molecular neurophysiology : biochemical reactions control the binding of receptors and the opening and closing of ion channels that gate the flow of ions through the cellular membrane. The conductance of a current at the cellular scale is a mean-field model of the actuation of a population of ions at a molecular scale. The macro-scale is the scale of circuit neurophysiology : the spatio-temporal electrical field models the activity of heterogenous neuronal tissues composed of distinct subpopulations of neurons involved in a specific brain function of interest. Macro-scale variables in neurophysiology are local field potentials, inferred from the total current recorded by an extracellular electrode over a specific tissue area.

\subsection{Outline}

The rest of the paper is organized in two sections. Section 2 revisits the central physiological concept of excitability as a particular combination of positive and negative feedback at the meso-scale of our network model. Section 3 illustrates the versatility of nodal control to 
shape the behavior of the network model anywhere from the nodal scale to the entire network scale. The exposition is deliberately non-technical and omits a lot of biological details to concentrate on the regulation principles of the considered circuits. The interested reader is referred to specific references for more details about their biological relevance.

\section{Mixed feedback amplification}

\subsection{Mean-field actuation models}

The conductance $g(\cdot)$ in the Ohmic law (2) models the mean-field, at a given meso-scale, of a population of actuators at the micro-scale. By nature, such a model is empirical and approximate. It is not meant to model the details of the micro-scale, but only to capture the extent to which the micro-actuators influence the current-voltage relationship at the mesoscale of interest. We write $g(\cdot)=\bar{g} S(\cdot)$, where $\bar{g}$ denotes the maximal conductance, and $S(\cdot)$ is an adimensional model of actuation with normalized output range $[0,1]$. We call $S(\cdot)$ a $S$-behavior. We briefly review basic models of the voltage dependence of a $S$-behavior.

The static properties of a $S$-behavior are illustrated in Figure 3:

- the $S$-behavior has two distinct read-outs: a continuous readout and a discrete readout; the continuous readout is a fine-grain readout whereas the discrete readout is a coarse-grain readout.

- the gain $\delta g=\frac{d S}{d V}(V) \delta V$ is localized in amplitude: it vanishes away from a restricted amplitude range and has a unimodal shape.

The unimodal profile of the gain provides a statistical interpretation of the mean-field model: each actuator in the population contributes to the mean-field gain in an infinitesimal range $\delta V$. The distribution of gains in the population is necessarily bell-shaped for a large population. The $S$-behavior is the cumulative distribution of the population. For a normally distributed population centered at $V=0$ and of variance $\sigma$, the cumulative distribution is the Gauss error function $S(V)=\operatorname{erf}(V)=\frac{1}{\pi} \int_{-V}^{V} e^{-x^{2}} d x$. Static models of $S$-behaviors are pervasive in science and engineering. An early appearance is in population studies, with the logistic model of Verhulst (13), which uses the sigmoid $S(V)=\frac{1}{1+e^{-V}}$. In (bio)chemistry, $S$-behaviors include the Hill activation function $S(V)=\frac{V^{\alpha}}{K^{\alpha}+V^{\alpha}}$. The parameter $K$ is the constant of half-activation and the parameter $\alpha$ controls the variance of the gain. In computer science, the hyperbolic tangent $S(V)=\tanh (V)$ is a frequent model of soft quantization arising in a population of agents with binary output. In electronic engineering, $S$-behaviors model amplifiers or transistors. In control theory, the piecewiselinear saturation models the finite sensitivity range of an actuator or sensor.

The static model of a $S$-behavior captures the restricted amplitude input range of the differential gain but does not capture its restricted temporal range. In nature and in engineering, $S$-behaviors have a limited bandwidth and a fading memory. The temporal dependence of the differential gain can be estimated empirically from the step response of the current to a voltage step perturbation (Figure 4). A unimodal step response corresponds to the localization of the differential gain in time, analogously to the localization in amplitude.

Modeling the fading property of a gain both in amplitude and temporal range is difficult. Static models only capture the amplitude localization. Linear time-invariant models only capture the temporal localization via the frequency dependence of the gain. Modeling the localization both in time and amplitude requires nonlinear dynamical models, which is a

8 Sepulchre, Drion, Franci 

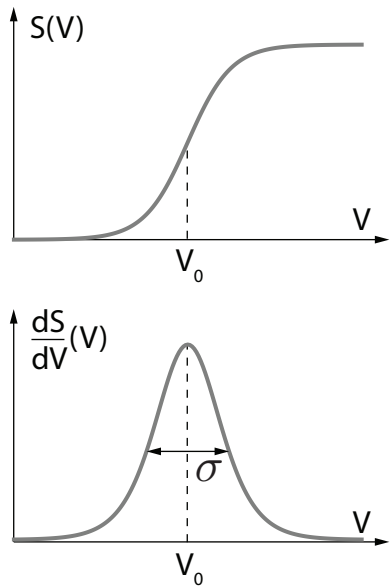

Figure 3

The static $S$-behavior of a mean-field model of actuation (a) and its (differential) gain (b). The mean-field behavior saturates away from a restricted amplitude range. It is the cumulative distribution of a population of devices only sensitive in a micro-range of amplitude. The unimodal gain models the first two moments (mean and variance) of a statistical distribution.

source of intractability in network models. This issue has been a pervasive challenge in amplifier modeling, see e.g. (14).

In neurophysiology, conductance-based modeling was pioneered by Hodgkin and Huxley (15). They designed the voltage-clamp experiment in which the voltage was clamped to a step change $\Delta V$ from the resting potential $V_{0}$ and the conductance $g(t)=\frac{\Delta I(t)}{\Delta V}$ was computed from the corresponding current response $\Delta I(t)$. Based on those step responses, they fitted the experimental data to a model that expresses the conductance as the product of an activation variable $m$ and an inactivation variable $h$ :

$$
\begin{aligned}
g=g(m, n) & =\bar{g} m^{\alpha} h^{\beta} \\
\tau_{m}(V) \dot{m} & =m+m_{\infty}(V) \\
\tau_{h}(V) \dot{h} & =-h+h_{\infty}(V)
\end{aligned}
$$

The activation function $m_{\infty}(V)$ is a static $S$-behavior, with a positive bell-shaped gain $m_{\infty}^{\prime}(V)$. The inactivation function $h_{\infty}(V)$ is the complement of a $S$-behavior, that is, $1-h_{\infty}(V)$ is a static $S$-behavior. The time-constant $\tau_{m}(V)$ is significantly smaller than the time-constant $\tau_{h}(V)$, meaning that the conductance first activates, then inactivates. The exponents $\alpha$ and $\beta$ are further parameters of the model. The dynamics of the activation and inactivation variables model the fast and slow lags.

For the sake of mathematical tractability, we follow the approach in (16). We assume an infinitesimal step change $\delta V$ around a fixed potential $V$ and we approximate the temporal properties of the differential conductance by extracting three snapshot local gains corresponding to the instantaneous response $\delta I^{0}$, the early response $\delta I^{f}$, and the late response $\delta I^{s}$ (Figure 4). The differential conductance is decomposed accordingly as

$$
\delta I=\delta g^{0} \delta V+\delta g^{f} \delta V+\delta g^{s} \delta V
$$

We assume a bell-shaped gain $\delta g^{f}(V)$ to model the early activation of the conductance and 

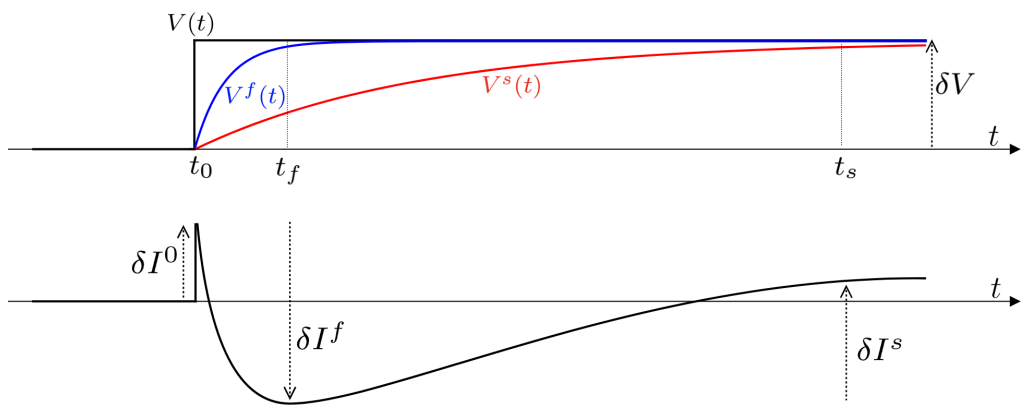

Figure 4

The temporal behavior of a differential conductance is approximated with three distinct snapshots: $\delta I^{0} \approx \delta g^{0}(V) \delta V$ for the instantaneous behavior, $\delta I^{f} \approx \delta g^{f}(V) \delta V$ for the early behavior and $\delta I^{s} \approx \delta g^{s}(V) \delta V$ for the late behavior.

a bell-shaped gain of opposite $\operatorname{sign} \delta g^{s}(V)$ to model its late inactivation. This corresponds to a conductance model of the general form

$$
g=g\left(V^{f}, V^{s}\right)=\bar{g} S^{f}\left(V^{f}\right)\left(1-S^{s}\left(V^{s}\right)\right)
$$

where the two variables $V^{f}$ and $V^{s}$ are assumed to lag behind $V$ with a fast and slow time-lag, respectively. The simplest lag model is of the form

$$
\begin{aligned}
\tau^{f} \dot{V}^{f} & =-V^{f}+V \\
\tau^{s} \dot{V}^{s} & =-V^{s}+V
\end{aligned}
$$

with $0<\tau^{f}<<\tau^{s}$ the time-constants of the fast and slow times-scales, respectively. In a quasi steady-state approximation, the conductance based models (5) and (3) are equivalent via the identification $S^{f}(V)=m_{\infty}^{\alpha}(V)$ and $S^{s}(V)=1-h_{\infty}^{\beta}(V)$.

The conductance model of Hodgkin and Huxley was purely empirical. Much later, with the development of molecular biology, the model was related to a microscopic description of the ion channel dynamics that gate the conductance. The model (3) was then interpreted as the mean-field evolution of a large population of discrete actuators modeled by a Markov process that flips their binary state with a voltage dependent probability (17).

\subsection{Nodal and network currents}

We consider Ohmic currents of four distinct types (Table 1). A first distinction is between passive and active currents. A current is said to be passive if its conductance is independent of the electrical field. The conductance of a passive current is a parameter $g(\cdot)=\bar{g}$. A current is said to be active if its conductance is voltage-dependent. A second distinction is between nodal and network currents. A current into a node is said to be nodal if it only depends on the nodal potential. It is called a network current if it depends on two distinct nodal potentials. With the reverse sign convention $I=-g(\cdot) \cdot(V-\tilde{V})$ for every network current into node $V$, the total nodal current into a node is equal to the total network current:

$$
I_{\text {nod }}=I_{\text {net }}
$$


This equation rephrases Kirchhofff's law that the total current $I_{n o d}-I_{n e t}$ into the node is zero. It also has the interpretation of a nodal balance between the internal and the external flows.

Conductances are assumed to be localized in a given amplitude and temporal range, as explained in the previous section. Nodal conductances are the conductances of nodal currents. Nodal control shapes the nodal behavior by modulating the maximal conductance of a nodal current. Network conductances are the conductances of network currents. Network control shapes the nodal interactions by modulating the maximal conductance of a network current.

Table 1 The four distinct types of Ohmic currents into node $k$. The parameter $E$ denotes an external constant potential (battery).

\begin{tabular}{|l|c|c|}
\hline Current types & Nodal & Network \\
\hline Passive & $\bar{g}\left(V_{k}-E\right)$ & $\bar{g}\left(V_{k}-V_{l}\right)$ \\
Active & $g\left(V_{k}^{f}, V_{k}^{s}\right)\left(V_{k}-E\right)$ & $g\left(V_{l}^{f}, V_{l}^{s}\right)\left(V_{k}-E\right)$ \\
\hline
\end{tabular}

\subsection{Excitability: a mixed feedback principle for signaling across scales}

In this section we revisit the basic property that a mixed-feedback structure is necessary and sufficient to make a passive node excitable. We show that excitability makes the nodal scale sensitive to the micro-scale and enables signaling to the macro-scale via a discrete event.

We consider a single node of voltage $V$ with three nodal currents: a passive current $I_{p}$ that determines the passive behavior of the node and two active currents $I_{1}$ and $I_{2}$ that shape the behavior with a mixture of positive and negative feedback :

$$
\begin{aligned}
& I_{p}=g_{p}\left(V-E_{p}\right) \\
& I_{1}=g_{1}\left(V^{f}, V^{s}\right)\left(V-E_{\max }\right) \\
& I_{2}=g_{2}\left(V^{s}\right)\left(V-E_{\min }\right)
\end{aligned}
$$

The nodal behavior determines a one-port circuit with external current $I_{n e t}$ and voltage $V$. Kirchhoff's law $I_{\text {net }}=I_{\text {nod }}=I_{p}+I_{1}+I_{2}$ rewrites as

$$
g_{\text {nod }} V=g_{p} E_{p}+g_{1} E_{\max }+g_{2} E_{\min }+I_{\text {net }}
$$

with $g_{\text {nod }}=g_{p}+g_{1}+g_{2}$. In the absence of a network current, the voltage is a convex combination of the three parameters $E_{p}, E_{\min }$, and $E_{\max }$ at any time. We assume $E_{\min }<$ $E_{p}<E_{\max }$, meaning that the current $I_{1}$ is always negative and that the current $I_{2}$ is always positive. In the terminology of electrophysiology, $I_{1}$ is an inward current, whereas $I_{2}$ is an outward current.

To determine how the nodal currents contribute to the feedback properties of the system, we linearize the circuit equations around a given voltage $V$ and separate the contributions 
of the circuit conductance in each time scale:

$$
\begin{aligned}
\delta I_{\text {net }} & =\delta I_{\text {nod }}^{0}+\delta I_{\text {nod }}^{f}+\delta I_{\text {nod }}^{s} \\
& =\frac{\partial I_{\text {nod }}}{\partial V} \delta V+\frac{\partial I_{\text {nod }}}{\partial V^{f}} \delta V+\frac{\partial I_{\text {nod }}}{\partial V^{s}} \delta V \\
& =g_{\text {nod }} \delta V+\delta g^{f}(V) \delta V+\delta g^{s}(V) \delta V
\end{aligned}
$$

with

$$
\begin{aligned}
& \delta g^{f}(V)=\frac{\partial g_{1}}{\partial V^{f}}(V, V)\left(V-E_{\max }\right) \leq 0 \\
& \delta g^{s}(V)=\frac{\partial g_{1}}{\partial V^{s}}(V, V)\left(V-E_{\max }\right)+g_{2}^{\prime}(V)\left(V-E_{\min }\right) \geq 0
\end{aligned}
$$

The local circuit behavior has a specific mixed-feedback structure (Figure 5): an open-loop process in the instantaneous time-scale, a positive feedback system in the fast time-scale, and a negative feedback system in the slow time-scale. The positive feedback is provided by the activation of an inward current. It is synonym of a negative conductance, or equivalently, a variation $\delta I^{f}$ of sign opposite to the variation $\delta V$ in the step response shown in Figure 4. The negative feedback is provided by the inactivation of the inward current and the activation of an outward current. Both contribute to a positive conductance, or equivalently, a variation $\delta I_{s}$ of the same sign as the variation $\delta V$ in the step response shown in Figure 4. The mixed feedback is first positive, then negative. This specific feedback structure makes the node excitable. An excitable behavior outputs discrete events, called spikes or action potentials, in response to continuous-time variations of the input signal (Figure 6). We refer the reader to (18) for a detailed discussion on excitable behaviors.

The positive feedback in the fast time-scale is responsible for a threshold around which the behavior is ultra-sensitive. For sub-threshold variations of the input current, the voltage response is passive, but beyond threshold, the voltage jumps to a value close to $E_{\max }$. The nodal behavior is all-or-none in the fast time scale: supra-threshold input variations trigger an output discrete switch. For an equilibrium behavior close to $E_{\min }$, the voltage switch spans almost the entire output voltage range $\left[E_{\min }, E_{\max }\right]$ of the circuit.

The negative feedback in the slow time-scale is responsible for a refractory time-interval following the switch. The voltage variable returns to equilbrium with a negative overshoot. During the refractory time-interval, the nodal behavior is infra-sensitive, that is, its sensitivity to an external perturbation is lower than the sensitivity of the open-loop behavior. The negative feedback turns the switch into a discrete event of finite duration. It is the slow time scale of the feedback system that controls the duration of the spike.

The excitable behavior that results from fast positive feedback and slow negative feedback is robust, tunable, and tractable. Those three properties are grounded in a sufficient separation of scales. The behavior is robust in that it only depends on qualitative properties of the actuation model, that is, the existence of a fine-scale amplitude and temporal voltage range where the positive feedback gain dominates and a coarse-scale amplitude and temporal voltage range where the negative feedback gain dominates. The behavior is tunable in that the two qualitative properties of excitability - the threshold and the refractoriness can be tuned by independent parameters. The maximal gain of the positive feedback loop determines the threshold. It is tuned by the maximal conductance of the inward current. The maximal gain of the negative feedback loop determines the refractoriness. It is tuned by the maximal conductance of the outward current. Finally, the behavior is tractable, in spite of being nonlinear and dynamic. Thanks to the time-scale separation, the fast switch and the slow refractoriness can be analyzed separately, for instance through fast-slow phase portrait analysis. In the absence of the time-scale separation, excitability is lost. Robustness, tunability, and tractability of the behavior progressively deteriorate as the time-scale 
separation decreases. In neurophysiology, the ratio between fast and slow time scales is rarely below 5 to 10 . This separation is also remarkably stable across different types of neurons.

The significance of excitability for the present paper is that an excitable node signals across scales. Near threshold, the meso-scale behavior is ultra-sensitive, that is, sensitive to smaller scales. This ultra-sensitivity is localized in a narrow amplitude and temporal range of the input signal. Away from this specific range, signals from the micro-scale are in the noise range of the passive node. But within the ultrasensitive range, signals from the microscale trigger a discrete event in the meso-scale. Excitability makes the meso-scale selectively sensitive to specific signals or ensemble of signals from the micro-scale. Likewise, the output signal of an excitable node is a sequence of discrete-events. Those discrete events can serve as the micro-actuators of a mean-field conductance at a larger scale. Collectively, many spikes control the mean-field gain of a macro-conductance. Because spikes have a specific amplitude and temporal range, the mean-field gain is necessarily localized in amplitude and temporal range. A population of spikes determines a mean-field conductance at the macroscale. As a mean-field of discrete events, the mean-field conductance defines a $S$-behavior for the next scale.

The role of mixed-feedback as the fundamental mechanism of excitability was first discovered by Hodgkin and Huxley. In their seminal work (15), they modeled excitability as the property of an electrical circuit consisting of a passive membrane, an inward (sodium) current with fast activation and slow inactivation, and an outward (potassium) current. (Their model only differs from the structure (8) by an additional capacitive current that is inessential for the message of the present paper). Their model was later shown by Fitzhugh (19) and Nagumo (20) to share the properties of negative resistance oscillators studied earlier by Van der Pol. A negative resistance device is an Ohmic element with a negative gain over some voltage range. The terminology is sometimes a source of confusion because it is not the resistance (or conductance) itself that is negative, but only its gain. The inward current $I_{1}$ in (8) has the interpretation of a negative resistance device when the fast timescale merges with the instantaneous time-scale, that is, $V^{f}=V$, and when the inactivation of the inward current is neglected (or merged with the activation of the outward current). The model of Hodgkin and Huxley is discussed in any textbook of neurophysiology, see e.g. (21). The presentation here leaves aside many physiological details in order to concentrate on the role of mixed-feedback in shaping the sensitivity properties of a passive node.

\subsection{A network of mixed-feedback amplifiers}

An excitable nodal behavior is determined by three distinct types of nodal currents: a passive current, and active currents that up-regulate or down-regulate the passive behavior. In the same way, the nodal interactions are determined by three types of network currents: passive network currents, and active network currents that are either excitatory or inhibitory.

In Figure 7, the resistive link between node 1 and node 2 denotes a network passive current :

$$
I_{12, p}=-I_{21, p}=-\bar{g}_{12}\left(V_{2}-V_{1}\right) .
$$

The link between node 1 and node 3 with an arrow symbol denotes an excitatory current into node 3 with a conductance that depends on the nodal potential $V_{1}$ :

$$
I_{13, E}=-g_{s y n}\left(V_{1}^{f}, V_{1}^{s}\right)\left(V_{3}-E_{s y n,+}\right)
$$




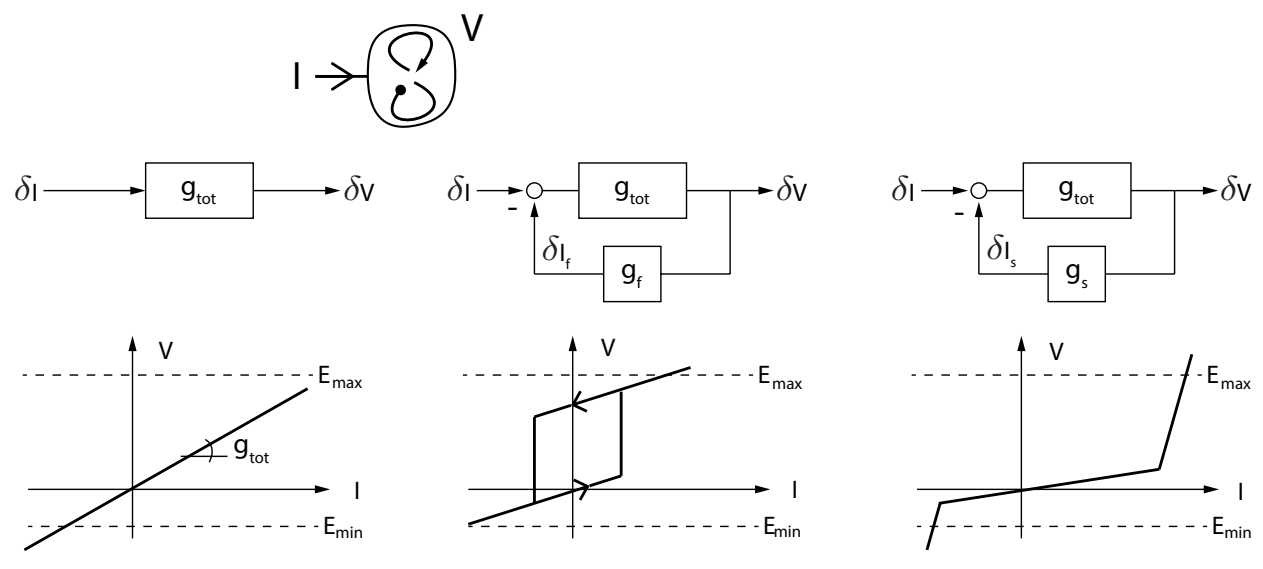

Figure 5

The behavior of a passive node with fast positive feedback and slow negative feedback is excitable: ultra-sensitive in the fast time-scale and infra-sensitive in the slow time-scale. The nodal conductances modulate the nodal behavior between an open-loop model (instantaneous time-scale), a positive feedback model (fast time-scale), and a negative feedback model (slow time-scale).

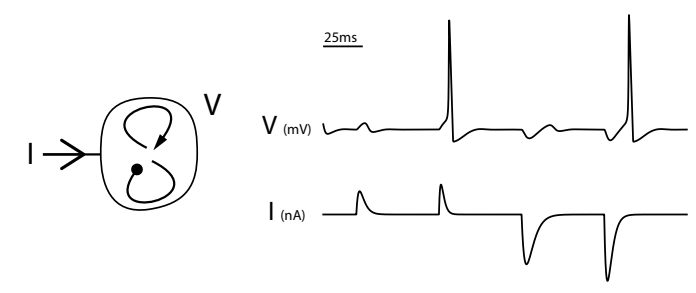

Figure 6

An excitable behavior converts the small variations of a continuous input signal into a sequence of discrete-events with a specific amplitude and temporal signature. Ultra-sensitivity in the fast time-scale endows the meso-scale with a threshold with respect to continuous variations at the micro-scale. Discrete-events at the meso-scale are the atoms of signaling at the macro-scale.

The current is called excitatory because the potential $E_{s y n,+}$ is higher than the excitability threshold of node 3 . For instance, the current is necessarily excitatory if $E_{\text {syn, }}=E_{\max }$ : a spike at node 1 will necessarily generate a positive current $I_{13, E}$, that will depolarize node 3 and therefore facilitate a spike at node 3 .

The link between node 2 and node 3 with a circle symbol denotes an inhibitory current into node 3 with a conductance that depends on the nodal potential $V_{2}$ :

$$
I_{23, I}=-g_{s y n}\left(V_{2}^{f}, V_{2}^{s}\right)\left(V_{3}-E_{s y n,-}\right)
$$

The current is called inhibitory because the potential $E_{\text {syn,- }}$ is lower than the excitability threshold of node 3 . For instance, the current is necessarily inhibitory if $E_{\text {syn,- }}=E_{\min }$ : a spike at node 2 will necessarily generate a negative current $I_{13, E}$, that will hyperpolarize node 3 .

Passive network currents model diffusion between the nodes. In neurophysiology, they 


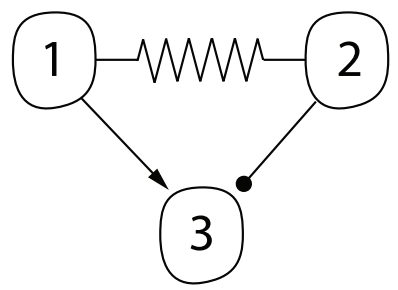

Figure 7

Interconnections between nodes are determined by three types of network currents. The resistor symbol denotes a passive current between node 1 and node 2 . The arrow symbol denotes an excitatory current into node 3 , with a conductance controlled by $V_{1}$. The circle symbol denotes an inhibitory current into node 3 , with a conductance controlled by $V_{2}$.

model gap junctions between neurons. Active network currents model synaptic interconnections between neurons. A frequent assumption in neuroscience is that the excitatory or inhibitory nature of a synaptic current is a property of the presynaptic node. Nodes are called excitatory when they control excitatory synaptic currents, and inhibitory when they control inhibitory synaptic currents. In Figure 7, the node 1 is excitatory whereas the node 2 is inhibitory.

The network currents above complete the description of a network model that includes the four distinct types of currents in Table 1. It is the presence of active nodal currents that distinguishes this model from many other models in the literature. The nodal active currents are essential to shape the nodal excitability.

In the systems and control literature, most dynamical network models only include the passive currents of Table 1. They consider passive nodal behaviors and passive interconnections. Passivity must here be understood in the generalized system-theoretic sense of behaviors that can only dissipate energy (22). The study of passive networks is a major topic of system theory (23). It focuses on equilibrium behaviors and the diffusive properties of passive interconnections. It is a core concept of the distributed control paradigm. Passive networks have a limited sensitivity across scales. The input-output sensitivity is expected to decrease with the size of the network, which limits the controllability properties at the network scale. Such limitations have become the focus of recent studies, see e.g. (24).

In computational neuroscience, the important class of rate models includes active network currents but do not includes active nodal currents. Neural networks with passive nodes and active interconnections originate in the seminal work of Wilson-Cowan (25), Amari (26), and Hopfield (27). The nodal behavior in those models is passive. It has the interpretation of a mean-field behavior of the cellular activity. The nodal dynamics does not model the spiking behavior of a single neuron but rather the mean-field spiking activity of a population of neurons. Rate neural network models have been seminal in identifying the network properties of specific network architectures encountered in neurophysiology. Rate models do not include the nodal feedback of nodal currents but do include the network feedback of any network loop of network currents. The network feedback can shape the sensitivity of a node very much like the nodal feedback. Our motivation to include nodal active currents in the network architecture is to highlight the distinct role of nodal control and network control in a multi-scale model.

The parallel types of nodal and network currents in our network architecture make it 
multi-scale and multi-resolution in nature: network currents at a finer scale become nodal currents at a coarser scale. In the same way, network conductances at the nodal scale become nodal conductances at the network scale. At the nodal scale, the behavior is an input-output relationship between $I_{n e t}$ and $V$. This input-output behavior is shaped by the nodal conductance, which acts as a feedback control. The input $I_{n e t}$ is shaped by the network conductance, which acts as a feedforward control.

\section{Shaping behaviors across scales}

First positive then negative mixed-feedback provides a basic feedback structure for signaling across scales. The second part of the paper will illustrate how controlling the nodal scale with this feedback structure can shape the network behavior at broader scales.

\subsection{Shaping a nodal behavior}

The mixed-feedback structure discussed in Section 2.3 endows the nodal behavior with a specific scale of excitability: the spike is a discrete event with a specific amplitude and temporal sensitivity range determined by the active nodal currents. A two-scale nodal behavior is obtained simply by duplicating the mixed-feedback structure. We consider a node with one passive current and four distinct active currents to shape two distinct mixedfeedback loops:

$$
\begin{aligned}
& I_{p}=g_{p} \cdot\left(V-E_{p}\right) \\
& I_{1}=g_{1}\left(V^{f}, V^{s}\right) \cdot\left(V-E_{\max }\right) \\
& I_{2}=g_{2}\left(V^{s}\right) \cdot\left(V-E_{\min }\right) \\
& I_{3}=g_{3}\left(V^{s}, V^{u s}\right) \cdot\left(V-E_{\max }\right) \\
& I_{4}=g_{4}\left(V^{u s}\right) \cdot\left(V-E_{\min }\right)
\end{aligned}
$$

The nodal circuit has now two distinct sources of positive feedback, each of which can create a distinct threshold. We assume that the activation ranges of the two inward currents $I_{1}$ and $I_{3}$ are distinct and overlapping, both in amplitude and in time: the activation range of $I_{3}$ is slower and lower than the activation range of $I_{1}$. The two outward currents $I_{2}$ and $I_{4}$ balance the two distinct ranges of positive feedback with two distinct ranges of negative feedback. They provide refractoriness in two distinct but overlapping ranges in amplitude and in time. Together, the four nodal currents shape the interlocked mixed-feedback of two distinct scales of excitability, with overlapping amplitude and temporal ranges. The resulting discrete event is a burst, which can be regarded as a spike of spikes (Figure 8).

Bursting is central to cellular neurophysiology. The four distinct types of nodal conductances can be identified in most neurons. Sodium and calcium currents are inward currents. The activation range of most calcium currents is slow and low with respect to the activation range of most sodium currents. Hence sodium activation is the most common source of fast positive feedback and calcium activation is a common source of slow positive feedback. Potassium and chloride currents provide most of the outward currents. Their activation ranges are widepspread in amplitude and in time, providing negative feedback in a continuum of ranges. Sometimes, it is the inactivation of an outward current that provides the slow positive feedback. A notable example is the A-type potassium current, which plays an important role in slow excitability (28).

The particular significance of bursting in the context of the present paper is that it endows the nodal behavior with two distinct modes of excitability, each associated with a 

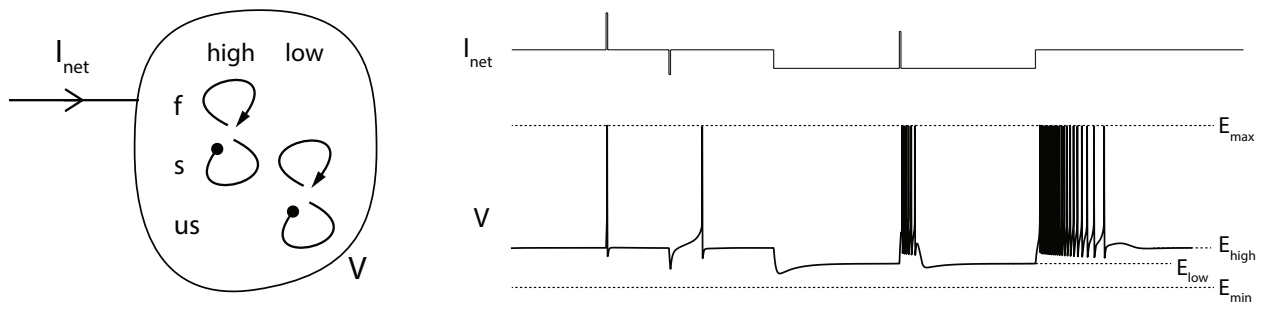

Figure 8

Two interlocked mixed-feedback loops endow the nodal behavior with two distinct modes of excitability. The equilibrium value of the node determines the excitability mode : the node is spike excitable around the equilibrium potential $V=E_{h i g h}$ and burst excitable around the equilibrium potential $V=E_{\text {low }}$. Each mixed-feedback loop endows the nodal behavior with a distinct scale of memory and signaling.

distinct scale. Spike excitability only signals in the high amplitude and frequency range; burst excitability simultaneously signals in a high and low amplitude and frequency ranges. The modulation between spike and burst excitability is central to the multiscale control illustrations of the next sections. It can take many different forms but the basic property is illustrated in Figure 8. The excitability mode drastically alters the input-output behavior of the node. A same input disturbance might trigger a spike or a burst, that is, generate a discrete event in two distinct scales. This is because of the distinct activation ranges of the two inward currents. The trajectories illustrated in Figure $\mathbf{8}$ are the result of a fast inward current that activates near $E_{h i g h}$ and a slow inward current that activates near $E_{\text {low }}$. Spike excitability is observed when the slow inward current is inactivated, whereas burst excitability is observed when the slow inward current is activated. A burst response is observed only when the voltage has remained low for long enough. The modulation between spike and burst excitability is central to the control of the nodal behavior. It can be achieved either by modulating the polarization of the node, that is, the balance of inward and outward currents at steady-state, or by modulating the maximal conductance of the slow inward current.

The behavior that results from two interlocked mixed-feedback loops is robust, tunable, and tractable. We refer the interested reader to a detailed analysis of those properties in (29). They are rooted in a sufficient separation of the amplitude and temporal ranges of the feedback gains : a fast time-scale and high voltage range where positive feedback dominates; an ultra-slow and low voltage range where negative feedback dominates; and an intermediate slow time-scale and middle voltage range where the instantaneous sign of the feedback loop depends on a balance between the negative feedback following the fast positive feedback and the positive feedback preceding the ultraslow negative feedback. The fast/high positive feedback determines the threshold of spike excitability. The ultra-slow/low negative feedback determines the refractoriness of burst excitability. The intermediate balance of positive and negative feedback determines the threshold of burst excitability and the refractoriness of spike excitability. The behavior is robust to the modeling details of the activation and inactivation properties because it is only the balance of feedback loops in a given time-scale and amplitude range that determines the qualitative properties (threshold and refractoriness) of the two excitability modes. The behavior is tunable because the activation range and maximal conductances of each current can be tuned to shape the 
bursting waveform in a broad range of amplitude and frequencies. The behavior is tractable because the attractor properties of a burster can be dissected in distinct time-scales. Singular perturbation analysis and singularity theory provide a complete classification of the possible combinations of spike and burst excitability that obey the hierarchy of the two mixed-feedback loops (30).

The regulation of the balance of positive and negative feedback in the intermediate scale of the behavior is of particular significance to shape the input-output sensitivity of the nodal behavior in distinct time scales. The continuous modulation between spiking and bursting is a core signaling mechanism in cellular neurophysiology $(31,32)$. The reader will notice that such a regulation is not possible in the absence of a nodal conductance providing positive feedback in the intermediate voltage amplitude and temporal range. We have shown the critical role of this distinct positive feedback in (29) for robustness and tunability of a bursting behavior. Surprisingly, ionic currents that provide slow positive feedback are often omitted in mathematical treatments of bursting (e.g. in the recent textbooks (33) and (34)).

\subsection{Shaping a motif behavior}

Modulating the excitability of a node has a profound influence on the behavior not only at the nodal scale, but also across scales. This is best exemplified in two interconnection motifs that have been extensively studied in the literature : the I-I motif and the E-I motif. The I-I motif is a symmetric inhibitory interconnection between two nodes, each controlling a network inhibitory current into the other node:

$$
\begin{aligned}
& I_{12, I}=g_{12}\left(V_{1}^{s}\right)\left(V_{2}-E_{\min }\right) \\
& I_{21, I}=g_{21}\left(V_{2}^{s}\right)\left(V_{1}-E_{\min }\right)
\end{aligned}
$$

The E-I motif is an asymmetric excitatory-inhibitory interconnection between two nodes, each controlling a network current of opposite sign into the other node:

$$
\begin{aligned}
I_{12, E} & =g_{12}\left(V_{1}^{f}\right)\left(V_{2}-E_{\max }\right) \\
I_{21, I} & =g_{12}\left(V_{2}^{s}\right)\left(V_{1}-E_{\min }\right)
\end{aligned}
$$

The I-I motif is central to the circuit neurophysiology of central pattern generators (35). It is a basic mechanism to generate autonomous circuit rhythms by interconnecting nodes that are burst excitable but do not necessarily oscillate in isolation. The mechanism is simple to understand from the input-output nodal behavior shown in Figure 8: A burst in neuron 1 induces a hyperpolarizing network current into node 2 . As a consequence, the termination of a burst in neuron 1 triggers a burst in neuron 2. The symmetric interconnection from neuron 2 to neuron 1 closes a network loop that can sustain an autonomous anti-phase rhythm in the circuit (Figure 9).

The E-I motif is central to the circuit neurophysiology of transient slow rhythms. The mechanism is again a simple consequence of the input-output nodal behavior shown in Figure 8. In the spike excitability mode, the two nodes do not interact and behave independently of each other. In the reported illustration (Figure 9), one node is repeatedly spiking while the other node is silent. In the burst excitability mode, the two nodes interact, triggering a new rhythm for the circuit (Figure 9).

The significance of both motifs for the present paper is that it is the nodal modulation of excitability that controls the circuit behavior. In both motifs, the circuit rhythm is 


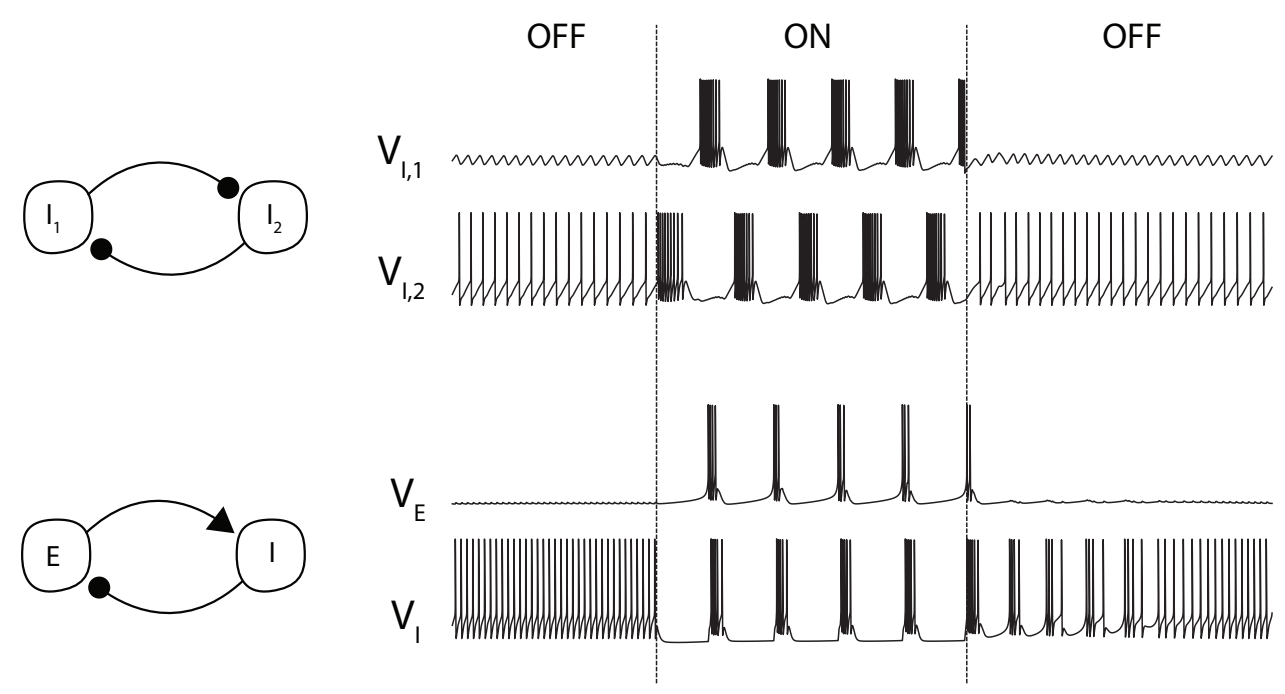

Figure 9

The influence of the nodal mode of excitability on two basic interconnection motifs. Both the I-I and E-I motif define a behavior at the circuit scale distinct from the behavior at a nodal scale.

The circuit behaviors are turned on and off by the modulating the excitability mode of the nodes.

turned on and off by the excitability mode of the nodes: the circuit rhythm is on when the nodes are burst excitable and off when the nodes are spike excitable. The resulting behavior is multi-scale because it can be modulated between a behavior at the nodal scale and a behavior at the motif scale. The control is at a nodal resolution, because the nodal excitability can be modulated locally, through local modulation of the maximal conductance of specific nodal currents.

The scale of the motif is of course not restricted to the interconnection of two cells. The interconnections can be between a population of excitatory nodes and a population of inhibitory nodes.

The multi-scale behavior of the elementary motifs discussed above is again robust, tunable, and tractable. We refer the interested reader to a detailed analysis of the I-I motif in (36) and a detailed analysis of the E-I motif in (37). Those properties are rooted in the separation of amplitude, temporal, and spatial scales. The separation of spatial scales is ensured by the assumption of weak nodal interconnection. The interconnection is weak if the nodal behavior is dominated by the feedback properties of nodal conductances. The nodal behavior of each neuron in Figure $\mathbf{9}$ is a sequence of spikes and bursts. Those are shaped by the nodal conductance, regardless of the interconnection topology. They define an input-output nodal behavior for each node, which is independent of the network arrangement. What differs in the E-I and I-I motif is only the sequence of discrete events in each node and the relative phase of similar events in the distinct nodes. Those properties are shaped by the network conductances. The separation of scales is fundamental to the multi-scale nature of the behavior. The behavior at a broader scale is a particular temporal arrangement of the discrete events that define the behavior at a finer scale.

The behavior of the motif is robust to the modeling details of the nodal and network conductances. This property is of fundamental importance to make the motif robust to 
the heterogeneity of the population when it designates the interconnection of populations rather than isolated nodes. The analysis in (36) (for the I-I motif) and in (37) (for the E-I motif) shows that the behavior illustrated in Figure 9 persists over a broad range of modeling parameters. In both papers, we highlight the critical role of the nodal slow positive feedback for this robustness. The two distinct positive feedbacks of each node determine two distinct types of discrete events, one for each mode of excitability. This discrete classification of the nodal behaviors is essential to the robustness of behaviors at a larger scale. The behavior of the motif is also tunable, precisely because the discrete classification of nodal behaviors persists over a broad range of parameters. Both the I-I and E-I circuit rhythms have distinct properties from the nodal behaviors. Those properties are determined by the phase relationships between the nodal discrete events. Those phase relationships can be tuned by modulating the activation ranges of the network currents, both in amplitude and time. Finally, the behavior of the motif is tractable because it can be inferred from the behavior of the nodes and from the circuit topology. The nodal behavior defines the discrete-events that compose the network behavior. The nodal interconnections constrain the phase relationships between the discrete events of interconnected nodes.

\subsection{Shaping a circuit behavior}

The nodal control of the I-I motif provides a shaping principle for circuits composed of excitable nodes interconnected by inhibitory synaptic currents. In neurophysiology, such circuits have long been associated to rhythmic functions such as breathing, chewing, swallowing, walking or heart beating $(38,39,40,41)$. We will briefly illustrate how nodal control shapes the behavior of the five-node circuit illustrated in Figure 10. We refer the interested reader to (42) for a detailed analysis of the circuit. The circuit architecture is inspired by the neuronal topology of the crab somatogastric ganglion (STG), which has served as a key experimental model to study the neuromodulation of rhythmic circuits over the past forty years (43). In experiments, two different rhythms coexist within the STG circuit. One corresponds to the fast pyloric rhythm, which is constantly active, whereas the second corresponds to the slow gastric mill rhythm, which can be turned on and off by afferent neuromodulatory inputs and neuromodulators. Each node in the circuit is a conductance-based model that includes seven different types of nodal currents. Those currents are not discussed in detail here but their conductances can be shown to shape the four distinct types of feedback discussed in Section 3.1. They endow each node with the two distinct excitability modes of a bursting node. In particular, the model includes two calcium currents that provide the critical source of slow/intermediate positive feedback necessary for burst excitability. Our local control parameter is the maximal conductance of those two currents, that can modulate the nodal excitability of each node between the two modes of excitability. The topology of the network is indicated in Figure 10. It includes passive currents between nodes 2,3 , and 4 , and a number of inhibitory network currents. Reciprocal inhibitory connections between nodes 1 and 2 and between 4 and 5 define two elementary I-I motifs, one for the fast rhythm between nodes 1 and 2, one for the slow rhythm between nodes 4 and 5 . The two distinct I-I motifs interact through the central node 3 .

The figure illustrates the different rhythms that can coexist in the circuit by controlling the excitability mode of the nodes. The significance of this nodal control is that the interconnections gains (and in particular the maximal conductances of the synaptic currents) 


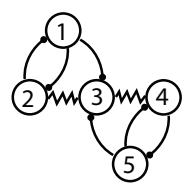

No rhythm
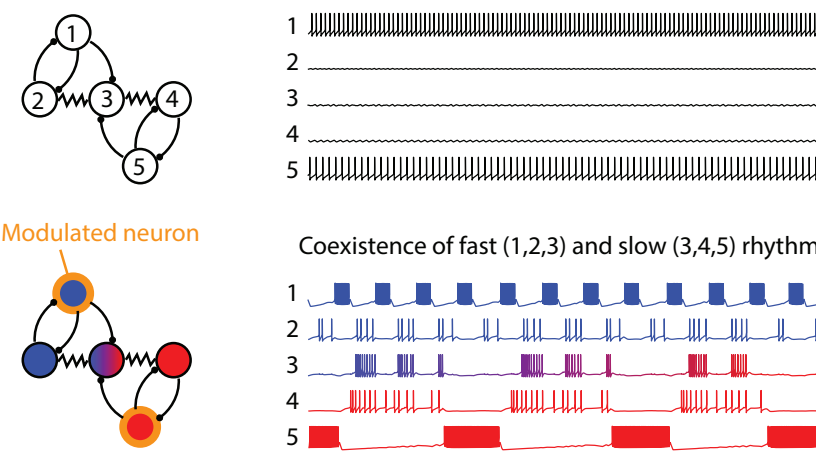

2

3

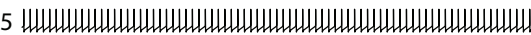

(1)

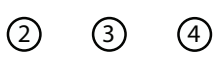

(5)
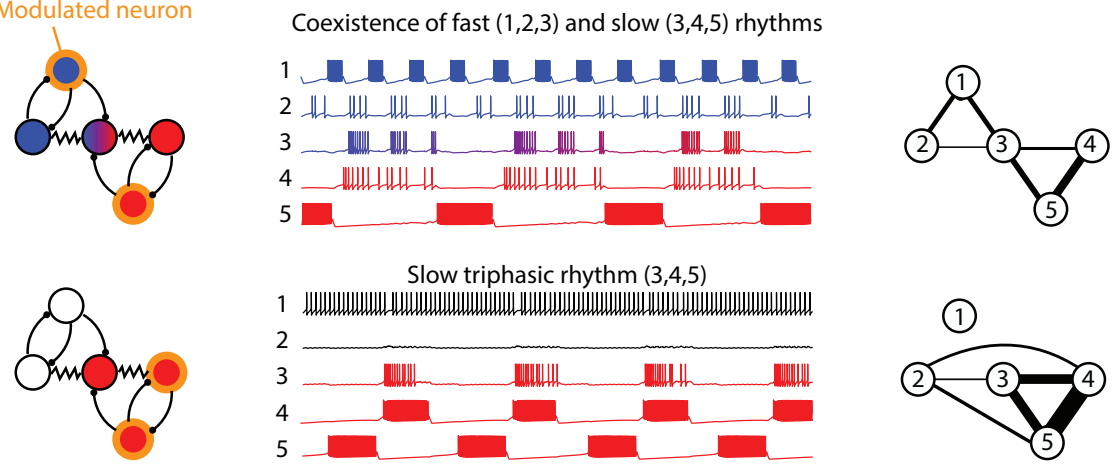

Slow triphasic rhythm $(3,4,5)$

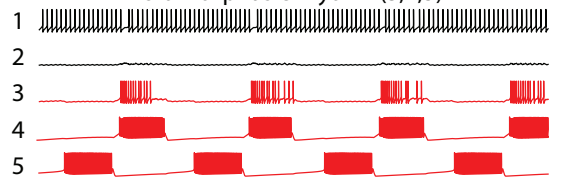

Fast triphasic rhythm $(1,2,3)$

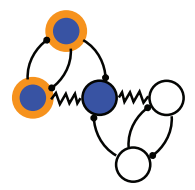

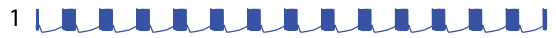
2 Lum

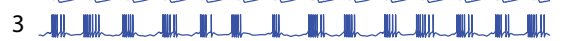

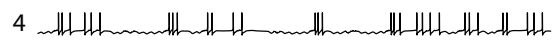

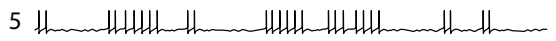

Global rhythm $(1,2,3,4,5)$
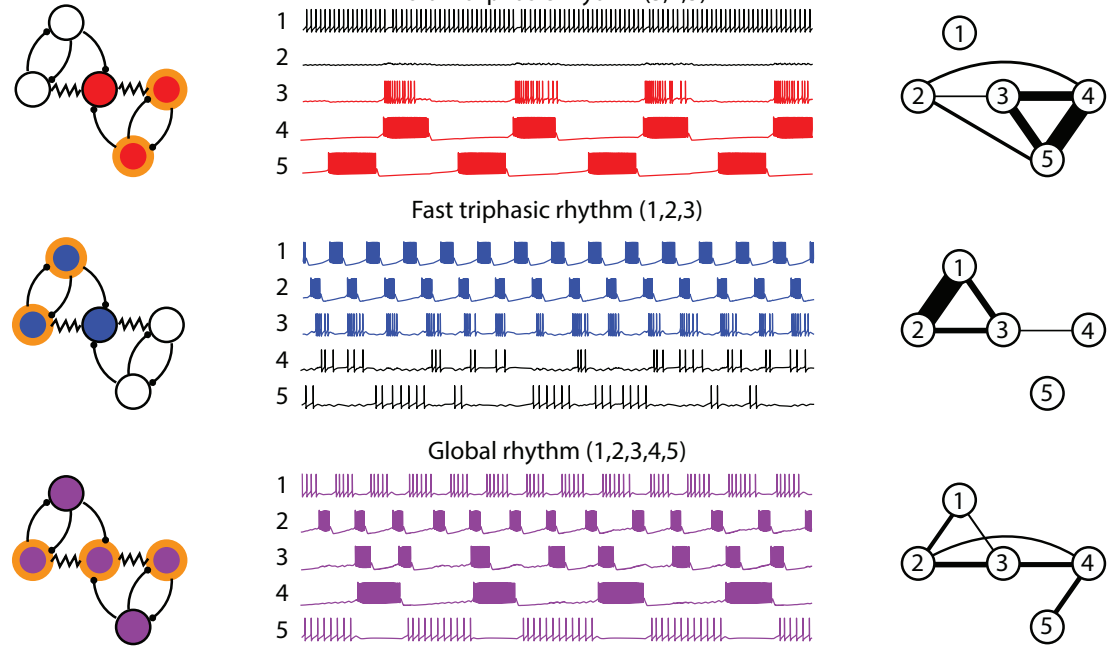

(5)

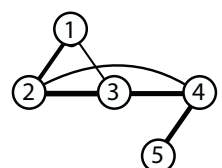

Figure 10

Nodal control of a circuit behavior with a fixed synaptic connectivity. Left, circuit connectivity diagrams. Filled circles represent neurons, which are numbered from 1 to 5 . Neurons with thick orange edges are controlled by neuromodulatory inputs that increase the maximal conductance of calcium currents. Center, membrane potential variations over time for neurons 1 to 5 (from top to bottom) in the different control configurations. Synaptic connections are identical in all cases. Neurons are colored in blue when they participate in the fast rhythm, in red when they participate in the slow rhythm, in purple when they participate in a global rhythm, and in black when they do not participate in the circuit rhythm. Right, functional connectome in the different control configurations.

are kept unchanged in the five circuit rhythms shown in Figure 10. The circuit behavior is modulated by nodal control rather than by network control. Nodal control modulates the functional connectivity of the network without changing the synaptic connectivity. Our message is that nodal control makes the circuit behavior robust, tunable, and tractable. The circuit behavior is easily inferred from the I-I motif behavior because the circuit topology only orchestrates the phase relationships between discrete events defined at a smaller scale. 
Those properties are very difficult to achieve when the circuit behavior is only tuned by the synaptic currents.

This example illustrates the role of nodal excitability in shaping a behavior across scales. This control principle is relevant to neurophysiology and illustrates the important function of neuromodulation as a complement to synaptic plasticity.

\subsection{Shaping a network behavior}

The control of the E-I motif provides a shaping principle for network states. Network states designate the spatio-temporal mean-field signature of specific discrete events at the network scale. In neuroscience, specific network states signal specific brain functions. Rapid control of network states has been reported to affect spatial attention in cortical circuits $(44,45)$, attention and arousal in the thalamus, and movement initiation in the subthalamic nucleus (46). The most studied example is probably the thalamo-cortical circuitry. The thalamus acts as a plastic relay between sensory systems, different subcortical areas and the cerebral cortex, by gating and modulating neuronal signal flow under the modulatory effect of cortical feedback $(47,48)$.

We briefly illustrate how nodal control shapes the spatiotemporal behavior of a network in the cartoon E-I network illustrated in Figure 11. The figure is taken from (37), which contains a detailed analysis of the model and its properties. Each node in the network again contains the four distinct types of feedback necessary to modulate the nodal behavior between spike excitability and burst excitability. The nodal control is through a neuromodulator that switches the node between its two modes of excitability by hyperpolarisation.

The network currents are of three distinct types:

$$
\begin{aligned}
I_{E I} & =g_{E}\left(V_{E}^{f}\right)\left(V_{I}-E_{\max }\right) \\
I_{I E, 1} & =g_{I, 1}\left(V_{I}^{s}\right)\left(V_{E}-E_{\min }^{1}\right) \\
I_{I E, 2} & =g_{I, 2}\left(V_{I}^{u s}\right)\left(V_{E}-E_{\min }\right)
\end{aligned}
$$

The excitatory current $I_{E I}$ models AMPA synaptic currents controlled by the excitatory neurons into the inhibitory neurons. The inhibitory currents $I_{I E, 1}$ and $I_{I E, 2}$ model GABA $A$ and $\mathrm{GABA}_{B}$ synaptic currents controlled by the inhibitory neurons into the excitatory neurons. $\mathrm{GABA}_{B}$ synapses are slower than $\mathrm{GABA}_{A}$ synapses, and $\mathrm{GABA}_{A}$ synapses have a reversal potential $E_{\min }^{1}$ slightly higher than $E_{\text {min }}$. The difference between the two different types of inhibitory currents is physiological but non essential for the present paper. Each pair of excitatory-inhibitory cells, as well as the entire populations of excitatory and inhibitory cells form a E-I motif with a qualitative behavior similar to the one discussed in Section 3.2.

Figure 11 illustrates how the nodal control of excitability can shape the spatiotemporal properties of the network. Very much like in the rhythmic circuit discussed in the preceding section, nodal control spatially modulates the functional connectivity of the network without changing the synaptic connectivity. Our message is that nodal control makes the network behavior robust, tunable, and tractable, at a nodal resolution. The network behavior is easily inferred from the E-I motif behavior because the nodal interconnections only orchestrate the spatial spread of localized nodal discrete events. 
A

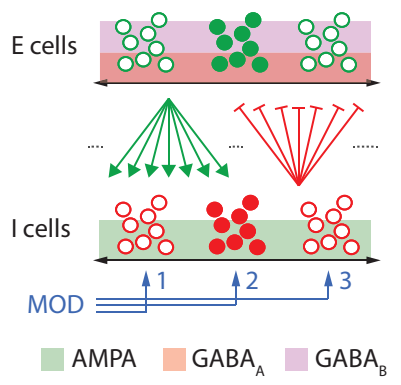

B

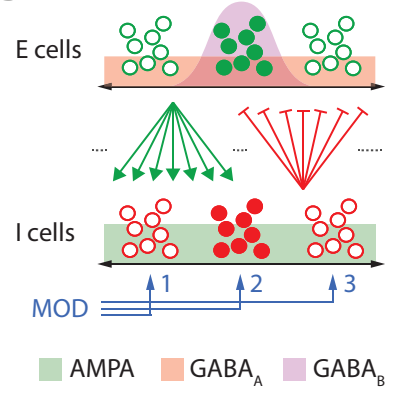

160 cells network (8 clusters) - LFP power
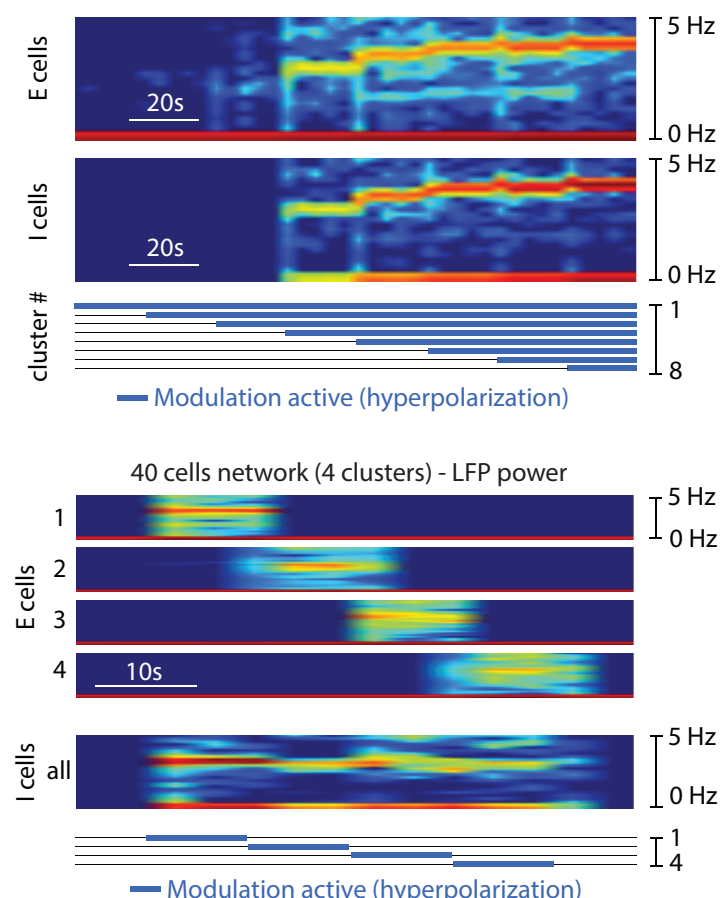

— Modulation active (hyperpolarization)

Figure 11

Nodal control shapes the spatiotemporal behavior of a E-I network at a cellular resolution. A, Left. Sketch of the spatial network. A one-dimensional array of 80 excitatory (E) cells is connected to a one-dimensional array of 80 inhibitory (I) cells. Inhibitory currents connect I cells to $E$ cells and are of two types $\left(\mathrm{GABA}_{A}\right.$ and $\left.\mathrm{GABA}_{B}\right)$. network currents are all-to-all from one population to the other population, with maximal conductances randomly distributed in a fixed range for each type of current. The nodal modulatory control (MOD) can be activated independently in 8 distinct spatial clusters A, right. Spectrogram of the local field potentials (LFP?s) of E cells (top) and I cells (bottom) with independent nodal control of 8 distinct spatial clusters. Blue traces at the bottom indicate the period during which the fast neuromodulator is active in a given cluster. Spatial localization of the nodal control affects the temporal frequency and amplitude range of the mean-field spectral power. B, Left. Sketch of the spatial network. A one-dimensional array of 20 excitatory (E) cells is connected to a one-dimensional array of 20 inhibitory (I) cells. Here, $\mathrm{GABA}_{B}$ connections are spatially localized, via a maximal conductance that decays exponentially along the spatial dimension. The nodal modulatory control (MOD) is activated independently in 4 distinct spatial clusters B, right. Spectrogram of the local field potentials (LFPs) of the 4 independently controlled spatial clusters of E cells (top) and I cells. Spatial localization of the nodal control and of the $\mathrm{GABA}_{B}$ currents only enables spatial tuning of the network mean-field, even though AMPA and $\mathrm{GABA}_{A}$ connections are all-to-all.

\section{Closing remarks}

A central paradigm of control theory is that feedback shapes sensitivity. How to extend this principle from a mono-scale feedback control loop to a multi-scale network control architecture remains a puzzle. This paper has focused on the role of nodal feedback control for network regulation. Nodal feedback control regulates nodal excitability, which is a specific 
type of ultra-sensitivity resulting from a combination of positive and negative feedback. We have shown that modulating nodal excitability can reconfigure a behavior at the large scale of a network with the tiny resolution of a node. This control principle is flexible, robust, and tractable provided that the actuation of each conductance is localized in the proper amplitude, temporal, and spatial ranges.

The material in this paper is primarily inspired from questions in neurophysiology. Understanding how neuromodulators can reconfigure the behavior of a neuronal circuit without altering its anatomy remains a central question in the analysis of brain functions. We have put aside many physiological details to concentrate on control principles that have generality beyond the applications of neuroscience. We hope that the potential of those principles across science and engineering stimulate novel developments in control theory. In particular, novel analysis and design tools are needed to cope with mixed-feedback control systems that behave away from equilibrium.

\section{SUMMARY POINTS}

1. Feedback shapes the sensitivity of an input-output behavior. Positive feedback is a source of ultra-sensitvity, memory, and discrete signaling. Negative feedback is a source of infra-sensitivity, linearity, and continuous signaling.

2. Excitability is a mixed-feedback mechanism. Excitability at a meso-scale shapes the (continuous) input sensitivity to finer scales and the (discrete) output signaling to coarser scales.

3. Nodal control of excitability is a versatile regulation mechanism across scales. It shapes the network scale of a spatio-temporal behavior at the resolution of the nodal scale.

\section{FUTURE ISSUES}

1. What is the potential of nodal feedback control in multi-scale control problems such as traffic control or artificial neural network regulation?

2. Linear control theory is a theory of sensitivity for negative feedback regulation of equilibrium behaviors. Is it possible to generalize linear control theory to a multiscale theory of sensitivity for mixed-feedback regulation of excitable behaviors?

3. Can nodal control advance the control principles of recurrent neural networks ?

\section{DISCLOSURE STATEMENT}

If the authors have noting to disclose, the following statement will be used: The authors are not aware of any affiliations, memberships, funding, or financial holdings that might be perceived as affecting the objectivity of this review.

\section{ACKNOWLEDGMENTS}

The research leading to these results has received funding from the European Research Council under the Advanced ERC Grant Agreement Switchlet n.670645 and from DGAPA- 
UNAM under the grant PAPIIT RA105518.

\section{References}

1. Olfati-Saber R, Murray R. 2004. Consensus problems in networks of agents with switching topology and time-delays. IEEE Transactions on Automatic Control 49:1520 - 1533

2. Sepulchre R. 2011. Consensus on Nonlinear Spaces. Annual Reviews in Control 35:56-64

3. Sarlette A, Sepulchre R. 2014. Control limitations from distributed sensing: Theory and extremely large telescope application. Automatica 50:421-430

4. Tucker G. 1972. The history of positive feedback: The oscillating audion, the regenerative receiver, and other applications up to around 1923. The Radio and Electronic Engineer 42:6980

5. Black H. 1934. Stabilised feedback amplifiers. Bell Labs Technical Journal 13:69-80

6. Thomas R. 1981. On the relation between the logical structure of systems and their ability to generate multiple steady states or sustained oscillation. Springer Ser. Synergetics 9:180?193

7. Tsai TYC, Choi YS, Ma W, Pomerening JR, Tang C, Ferrell JE. 2008. Robust, tunable biological oscillations from interlinked positive and negative feedback loops. Science (New York, N.Y.) $321: 126-129$

8. Mitrophanov AY, Groisman E. 2008. Positive feedback in cellular control systems. Bioessays 30:542?555

9. Smolen P, Baxter D, Byrne J. 2001. Modeling circadian oscillations with interlocking positive and negative feedback loops. Journal of Neuroscience 21:6644-6656

10. Astrom K, Murray R. 2008. Feedback systems: an introduction for scientists and engineers. Princeton university press

11. Nyquist H. 1932. Regeneration theory. Bell Labs Technical Journal 11:126-147

12. Koch C. 2004. Biophysics of computation: information processing in single neurons. Oxford university press

13. 2011. Verhulst and the logistic equation (1838). In A Short History of Mathematical Population Dynamics. Springer-Verlag

14. Boyd S, Chua LO. 1985. Fading memory and the problem of approximating nonlinear operators with volterra series. IEEE Transactions on Circuits and Systems 32:1150-1171

15. Hodgkin AL, Huxley AF. 1952. A quantitative description of membrane current and its application to conduction and excitation in nerve. The Journal of physiology 117:500-544

16. Drion G, Franci A, Dethier J.and Sepulchre R. 2015. Dynamic input conductances shape neuronal spiking. eneuro 2

17. Destexhe A, Mainen ZF, Sejnowski TJ. 1998. Kinetic models of synaptic transmission. In Methods in Neuronal Modeling. MIT Press

18. Sepulchre R, Drion G, Franci A. 2018. Excitable behaviors. In Emerging Applications of Control and Systems Theory. Springer-Verlag

19. FitzHugh R. 1961. Impulses and physiological states in theoretical models of nerve membrane. Biophysical journal 1:445

20. Nagumo J, Arimoto S, Yoshizawa S. 1962. An active pulse transmission line simulating nerve axon. Proceedings of the IRE 50:2061-2070

21. Byrne JH, Heidelberger R, Waxham MN. 2014. From molecules to networks: an introduction to cellular and molecular neuroscience. Academic Press

22. Willems J. 1972. Dissipative dynamical systems part i: General theory. Arch. Rational Mech. Anal. 45:321-351

23. van der Schaft AJ, Maschke BM. 2013. Port-hamiltonian systems on graphs. SIAM J. Control Optimisation 51:906?937

24. Pasqualetti F, Zampieri S, Bullo F. 2014. Controllability metrics, limitations and algorithms for complex networks. IEEE Transactions on Control of Network Systems 1:40-52 
25. Wilson HR, Cowan JD. 1972. Excitatory and inhibitory interactions in localized populations of model neurons. Biophys J 12:1-24

26. Amari SI. 1977. Dynamics of pattern formation in lateral-inhibition type neural fields. Biological cybernetics $27: 77-87$

27. Hopfield JJ. 1984. Neurons with graded response have collective computational properties like those of two-state neurons. Proceedings of the National Academy of Sciences 81:3088-92

28. Drion G, O'Leary T, Marder E. 2015. Ion channel degeneracy enables robust and tunable neuronal firing rates. Proceedings of the National Academy of Sciences 112:E5361-E5370

29. Franci A, Drion G, Sepulchre R. 2017. Robust and tunable bursting requires slow positive feedback. Journal of neurophysiology 119:1222-1234

30. Franci A, Drion G, Sepulchre R. 2014. Modeling the modulation of neuronal bursting: a singularity theory approach. SIAM Journal on Applied Dynamical Systems 13:798-829

31. Sherman SM. 2001. Tonic and burst firing: dual modes of thalamocortical relay. Trends Neurosci 24:122-6

32. Krahe R, Gabbiani F. 2004. Burst firing in sensory systems. Nature Reviews Neuroscience $5: 13-23$

33. Izhikevich EM. 2007. Dynamical systems in neuroscience. MIT press

34. Ermentrout B, Terman DH. 2010. Foundations of mathematical neuroscience. Springer

35. Marder E, Calabrese RL. 1996. Principles of rhythmic motor pattern generation. Physiol. Rev. 76:687-717

36. Dethier J, Drion G, Franci A, Sepulchre R. 2015. A positive feedback at the cellular level promotes robustness and modulation at the circuit level. Journal of neurophysiology 114:24722484

37. Drion G, Dethier J, Franci A, Sepulchre R. 2018. Switchable slow cellular conductances determine robustness and tunability of network states. PLoS Comput Biol 14:e1006125

38. Gordon I, Whelan P. 2006. Monoaminergic control of cauda-equina-evoked locomotion in the neonatal mouse spinal cord. J Neurophysiol. 96:3122-3129

39. Harris-Warrick R, Cohen A. 1985. Serotonin modulates the central pattern generator for locomotion in the isolated lamprey spinal cord. J Exp Biol. 116:27-46

40. Liu J, Akay T, Hedlund P, Pearson K, Jordan L. 2009. Spinal 5-ht7 receptors are critical for alternating activity during locomotion: in vitro neonatal and in vivo adult studies using 5-ht7 receptor knockout mice. J Neurophysiol. 102:337-348

41. Jordan L, Slawinska U. 2011. Modulation of rhythmic movement: Control of coordination, vol. 188 of Progress in Brain Research. Elsevier, 181 - 195

42. Drion G, Franci A, Sepulchre R. 2018. Cellular switches orchestrate rhythmic circuits. Biological Cybernetics ?:?

43. Marder E, Bucher D. 2007. Understanding circuit dynamics using the stomatogastric nervous system of lobsters and crabs. Annu. Rev. Physiol. 69:291-316

44. McCormick DA, Bal T. 1997. Sleep and arousal: thalamocortical mechanisms. Annu Rev Neurosci 20:185-215

45. McCormick DA NM. 2014. Editorial overview: neuromodulation: tuning the properties of neurons, networks and behavior. Curr Opin Neurobiol 29:iv-vii

46. AA K, D W, A K, P L, M H, et al. 2004. Event-related beta desynchronization in human subthalamic nucleus correlates with motor performance. Brain 137:735-746

47. Sherman SM, Guillery RW. 2006. Exploring the Thalamus and Its Role in Cortical Function. MIT Press, 2nd ed.

48. Sherman SM. 2012. Thalamocortical interactions. Current opinion in neurobiology 22:575-579 